\title{
Microcephaly-polymicrogyria-corpus callosum agenesis syndrome
}

INSERM

\section{Source}

INSERM. (1999). Orphanet: an online rare disease and orphan drug data base.

Microcephaly-polymicrogyria-corpus callosum agenesis syndrome. ORPHA:171703

Microcephaly-polymicrogyria-corpus callosum agenesis syndrome is a rare, genetic,

central nervous system malformation syndrome characterized by marked prenatal-onset

microcephaly, severe motor delay with hypotonia, bilateral polymicrogyria, corpus

callosum agenesis, ventricular dilation, small cerebellum and early lethality. 